\section{DERIVATION OF ELASTIC FRACTURE TOUGHNESS FOR DUCTILE METAL PIPES WITH CIRCUMFERENTIAL EXTERNAL CRACKS UNDER COMBINED TENSION AND BENDING}

\author{
Chun-Qing $\mathrm{Li}^{1}{ }^{1,}$, Guoyang $\mathrm{Fu}^{1}$, Wei Yang ${ }^{2}$ and Shangtong $\mathrm{Yang}^{3}$ \\ ${ }^{1}$ School of Engineering, RMIT University, Melbourne, Australia \\ ${ }^{2}$ College of Engineering and Science, Victoria University, Melbourne, 8001, Australia \\ ${ }^{3}$ Department of Civil \& Environmental Engineering, University of Strathclyde, Glasgow, UK
}

\title{
ABSTRACT
}

Linear elastic fracture mechanics has been widely employed for fracture analysis of cracked pipes. For ductile metal pipes, the existence of plasticity eases the stress concentration at the crack front, which increases the fracture toughness of the pipe. Therefore, when using linear elastic fracture mechanics to predict the fracture failure of ductile pipes, the plastic portion of the fracture toughness should be excluded. This paper intends to derive an analytical model of elastic fracture toughness for ductile metal pipes with circumferential external surface cracks under combined axial tension and bending. The derived elastic fracture toughness is a function of crack geometry, material properties and loading conditions of the cracked pipe. The significance of the derived model is that the well established linear elastic fracture mechanics can be used for ductile materials in predicting the fracture failure. It is found in the paper that, the elastic fracture toughness increases with the increase of the internal pressure of the pipe and that an increase in fracture toughness and yield strength of the pipe materials will result in a more ductile and brittle pipe failure respectively. The derived analytical model enables more accurate prediction of fracture failure of ductile metal pipes with circumferential external cracks.

\section{KEYWORDS}

Fracture Toughness; Ductile material; Circumferential Crack; Plasticity; Stress Intensity Factor.

*Corresponding author: Professor Chun-Qing Li, Email: chunqing.li@rmit.edu.au 
31 Pipelines are essential infrastructure that play a pivotal role in a nation's economy, prosperity, health, environment, social well-being and quality of life. Various materials have been used to make pipes, a significant portion of which are ductile metals, e.g. steel. Due to their long term service and exposure to corrosive environment, aging and deterioration of metal pipes have resulted in failures well before the end of intended design life.

Through investigation, it has been found that most metal pipe failures are of fracture type, caused by the propagation of a surface crack or defect (Cabral and Kimber 1997; Rajeev et al. 2014). For cracked ductile metal pipes under applied loading, the crack front yields before the stress intensity factor reaches its critical value. This yielding eases the stress concentration at the crack front, and as a result, the fracture resistance, known as fracture toughness, increases. It follows that for ductile materials, fracture toughness of ductile materials should consist of elastic and plastic portions, with the latter contributed by the plasticity of the material. As is well known, most of fracture analysis is based on linear elastic fracture mechanics (LEFM), in which the materials do not reach the plastic stage. In order to make use of LEFM for ductile materials, the plastic portion of fracture toughness should be excluded from the overall or total fracture toughness. Otherwise the value of fracture toughness will be overestimated, resulting in an underestimated probability of pipe failure.

Extensive research has been conducted on calculating the stress intensity factors for surface cracks in pipes (e.g. Raju and Newman 1982; 1986; Mettu et al. 1992; Kou and Burdekin 2006; Li and Yang 2012). However, stress intensity factors thus obtained are only applicable to elastic materials and plastic materials under small scale yielding conditions (Anderson 1991). For ductile materials with large scale yielding, elastic-plastic parameters such as $J$ integral (Rice 1968) and Crack Tip Opening Displacement (Burdekin and Stone 1966) have to be used. As such, nonlinear finite element fracture analyses are often resorted to for investigating the behaviours of 
cracked pipes made of ductile materials (e.g. Jayadevan 2004; Zhang et al. 2015), which requires more effort compared with elastic analyses. Further developments of the fracture mechanics based assessment led to the failure assessment diagrams, which are the most widely used methodology for elastic-plastic fracture analysis of structural components (Ainsworth 2003). Dowling and Townley (1975) identified two principal failure criteria, i.e., brittle fracture and plastic collapse, which is an essential step towards the development of failure assessment diagrams. These two criteria were then employed by Harrison et al. (1976) to define failure assessment diagram based on a modified strip yield model. Milne et al. (1988) presented detailed procedures for structural integrity assessment of ductile metal pipes with cracks, in which, failure assessment curves can be created based on the reference stress approach or elastic-plastic $J$ integral analysis. Using their assessment method, the conditions of cracked pipes can be assessed by checking against the failure curves, with the consideration of both fracture and plasticity of pipe materials.

For fracture analysis of pipes made of ductile materials, if the portion of the fracture resistance within the elastic range can be determined, the widely available results based on linear elastic fracture mechanics can be readily applied. In this way, the simple criterion of stress intensity factor used in LEFM still applies. Literature review (see references) suggests that very little research has been carried out on distinguishing the elastic and plastic potions of fracture toughness for ductile materials. Yang et al. (2016) can be one of the first researchers that proposed an analytical model of elastic fracture toughness for steel pipes with internal cracks but that study is limited to internal longitudinal cracks under a single loading, i.e., internal pressure.

As is well known, the stress intensity factors are geometry and stress (loading) dependent. Although fracture toughness is a material property, it is also affected by geometry and loading once the material reaches plastic stage. Since pipes are frequently subjected to axial tension and bending in service, circumferential cracks often occur in pipes. As different cracks and loading 
conditions may incur different failure modes, different analyses are required in deriving the elastic fracture toughness. This gives rise to the need for the present paper.

This paper aims to derive an analytical model of elastic fracture toughness for circumferentially cracked ductile pipes under combined axial tension and bending loads. The elastic fracture toughness in the derived model is a function of the geometry and material properties of the cracked pipe and applied loading. After verification of the derived model, parametric studies are conducted to investigate the effect of some key parameters on the elastic fracture toughness. The merit of the derived model is that it allows the use of extensive results based on linear elastic fracture toughness by engineers and asset managers for both design and assessment of ductile metal pipes, which can prevent future failures of pipes.

\section{FORMULATION OF ELASTIC FAILURE TOUGHNESS}

It is known that a cracked brittle pipe fails when the stress intensity factor $K_{I}$ at the crack front exceeds the fracture toughness $K_{I C}$. For pipes made of ductile materials, plasticity develops at the crack front, which eases the stress concentration at the crack front. As a result, the fracture toughness increases. Consequently, the total fracture toughness should consist of two parts as follows

$$
K_{I C}=K_{I C}^{e}+K_{I C}^{p}
$$

where $K_{I C}^{e}$ is the elastic portion of the total fracture toughness $K_{I C}$, termed elastic fracture toughness, whereas $K_{I C}^{p}$ is the plastic portion, referred to as plastic fracture toughness in this paper. To enable the use of LEFM for fracture analysis of ductile materials, $K_{I C}^{e}$ can be extracted from the total fracture toughness and denoted as follows

$$
K_{I C}^{e}=\alpha K_{I c}
$$


102 where $\alpha$ is defined as the ratio of the elastic fracture toughness to the total fracture toughness. By

103 subtracting the elastic fracture toughness from Eq. (1), the plastic fracture toughness $K_{I C}^{p}$ can be 104 determined as follows

$$
K_{I C}^{p}=(1-\alpha) K_{I c}
$$

106 With this separation, the following failure criteria in LEFM can still be used to assess the fracture 107 conditions of ductile metal pipes

$$
K_{I} \leq K_{I C}^{e}
$$

Therefore, the key to the application of LEFM for ductile materials is to determine $\alpha$.

110 For ductile metal pipes, failures often occur due to the interaction between two principal failure 111 modes, i.e., brittle fracture and plastic collapse. Two parameters have been employed to quantify 112 the two failure modes separately in the structural integrity assessment of cracked pipes as follows 113 (Milne et al. 1988)

$$
\begin{gathered}
K_{r}=K_{I} / K_{I C} \\
L_{r}=P / P_{L}
\end{gathered}
$$

115 where $P$ is the applied loading and $P_{L}$ is the corresponding plastic load limit of the cracked pipes.

116 Based on experimental results, a relationship between $K_{r}$ and $L_{r}$ has been developed as follows 117 (SINTAP 1999)

$$
K_{r}=\left(1+0.5 L_{r}^{2}\right)^{-0.5}\left[0.3+0.7 \exp \left(-0.65 L_{r}^{6}\right)\right]
$$

119 where the maximum value of $L_{r}$ is defined as $\bar{\sigma} / \sigma_{y}, \sigma_{y}$ is the yield stress and $\bar{\sigma}$ is the uniaxial 120 flow stress, calculated as the average of the yield and ultimate tensile strengths. Equation (6) has 121 been widely used by researchers and practitioners as the failure assessment of cracked structures. 
122 It needs to be noted that Equation (6) is the only relation between $K_{r}$ and $L_{r}$ as shown in literature

123 (SINTAP 1999). Thus Equation (6) is employed as the basis for the derivation of the elastic 124 fracture toughness for circumferentially cracked ductile metal pipes.

\section{DERIVATION OF ELASTIC FRACTURE TOUGHNESS}

126

Equation (6) establishes the relation among stress intensity factors $K_{I}$, fracture toughness $K_{I C}$, applied external loading $P$ and plastic load limits $P_{L}$ for cracked pipes. The Mode I fracture toughness of a certain metal $K_{I C}$ can be determined using ASTM standard testing method (ASTM E1820-01). In this section, the formula for stress intensity factor $K_{I}$ assuming linear elastic material are first derived by regression analyses based on finite element results. Then the analytical solution to plastic limit load $P_{L}$ developed by Kim et al. (2003) is employed for circumferentially cracked pipes under combined axial tension and bending. Finally, the analytical model of elastic fracture toughness is developed with the consideration of different combinations of axial tension and bending.

Formula for Stress Intensity Factor (SIF). As it is known, the axial tension and bending forces only result in the opening mode (Mode I) fracture with a circumferential external surface crack in pipes (Figure 1). The Mode I SIFs for any point along the crack front can be expressed as follows (Raju and Newman 1982)

$$
K_{I}=\sigma \sqrt{\pi a / Q} F_{I}\left(a / d, a / c, d / R_{i}, \varphi\right)
$$

where $\sigma$ is the applied stress, which can be $\sigma_{a}$ induced by axial tension $N$ or $\sigma_{b}$ induced by bending $M, a$ is the crack depth, $Q$ is the shape factor for an ellipse, $c$ is half crack length, $d$ and $R_{i}$ are the thickness and internal radius of the pipe respectively, $\varphi$ is used to define the position along the semi-elliptical crack and $F_{I}$ is the influence coefficient for Mode I fracture, as a function of the above parameters. 
145 In order to obtain a general formula for pipes with circumferential surface cracks, three146 dimensional finite element analyses are performed using ABAQUS (2011) to obtain SIFs for a 147 wide range of crack and pipe geometries. The energy based $J$-integral method, which is path148 independent and can produce accurate results with relatively coarse meshes, is employed. In 149 addition, the meshing technique with mixed quadratic hexahedron and tetrahedron elements in $\mathrm{Li}$ 150 et al. (2016) is adopted. Due to the free surface effect (Pook 1993), stress intensity factors at the 151 surface points are estimated by extrapolation from the results close to the surface. As the critical 152 values of SIFs occur either at the surface or deepest point along the crack front, only the results 153 from these two locations are studied. Based on the finite element results, formulae of the 154 influence coefficients of SIFs for circumferential cracks in pipes under axial tension and bending 155 respectively are obtained by performing non-linear regression (MathWorks 2013) as follows

$$
F_{I}(\cdot)=\left\{h_{1}+h_{2}(a / c)+\left[h_{3}+h_{4}(a / c)+h_{5}(a / c)^{2}\right](a / d)^{2}+\left[h_{6}+h_{7}(a / c)+h_{8}(a / c)^{2}\right]\right.
$$

$$
\left.(a / d)^{4}\right\} \exp \left(h_{9}\left(d / R_{i}\right)\right)
$$

158 where the values of coefficient $h_{i}(i=1,2, \cdots, 9)$ are listed in Table 1 for different loadings and 159 geometries ( $a / c$ ranges from $0.4-1.5, a / d$ ranges from $0.2-0.8$, and $d / R_{i}$ ranges from $0.1-1$ ). 160 Equation (8) is within $\mp 6 \%$ of the finite element results, which is sufficiently accurate for 161 deriving $K_{I C}^{e}$.

162 For pipes under a combination of axial tension and bending, the SIFs can be calculated based on 163 the principle of superposition (Anderson 1991)

$$
K_{I}=\sqrt{\pi a / Q}\left[\sigma_{a} F_{I}(N)+\sigma_{b} F_{I}(M)\right]
$$
respectively. 
167 For pipes with a circumferential crack under axial tension $N$ and bending $M$, the uniform axial stress $\sigma_{a}$ and maximum bending stress $\sigma_{b}$ can be represented as follows

$$
\begin{aligned}
\sigma_{a} & =\frac{N}{\pi\left(R_{o}^{2}-R_{i}^{2}\right)} \\
\sigma_{b} & =\frac{4 M R_{o}}{\pi\left(R_{o}^{4}-R_{i}^{4}\right)}
\end{aligned}
$$

170

where

$$
N_{L}^{\prime}=2 \pi \sigma_{y} R_{m} d\left[1-\frac{a}{d} \frac{\theta}{4}-\frac{2 \sin ^{-1}\left(\frac{a f(\theta)}{d 2 \theta}\right)}{\pi}\right]
$$

Substituting Equation (10) into Equation (9) yields the following

$$
K_{I}=\sqrt{\pi a / Q}\left[\frac{N}{\pi\left(R_{o}^{2}-R_{i}^{2}\right)} F_{I}(N)+\frac{4 M R_{o}}{\pi\left(R_{o}^{4}-R_{i}^{4}\right)} F_{I}(M)\right]
$$

$$
\frac{N}{2 \pi \sigma_{y} R_{m} d} \frac{N_{L}^{\prime}}{N_{L}}=1-\frac{a}{d} \frac{\theta}{4}-\frac{2 \sin ^{-1}\left(\frac{M}{4 \sigma_{y} R_{m}^{2} d} \frac{M_{L}^{\prime}}{M_{L}}+\frac{a f(\theta)}{d 2 \theta}\right)}{\pi}
$$

$$
M_{L}^{\prime}=4 \sigma_{y} R_{m}^{2} d\left[\cos \left(\frac{\pi}{8} \frac{a}{d} \theta\right)-\frac{a}{d} \frac{f(\theta)}{2 \theta}\right]
$$

$$
f(\theta)=0.7854 \theta^{2}-0.0982 \theta^{4}+0.0041 \theta^{6}-0.000085 \theta^{8}
$$

Plastic Limit Load. The majority of existing plastic load limits for pipes have been proposed either empirically based on piping testing data or analytically based on a simple yield criterion. These solutions tend to underestimate the actual limit load with unknown conservatism (Kim et al. 2002). For engineering assessment of cracked pipes, it is important to accurately determine the plastic limit loads. In this study, the analytical solution to the plastic load limit for pipe under combined axial tension and bending is employed. The solution was derived by Kim et al. (2003) based on stress fields equilibrium and improved by finite element limit analyses as follows

$$
\theta=\frac{c}{R_{m}}
$$


$N_{L}$ is the plastic limit load for axial tension and given by

185

$$
N_{L}=2 \pi \sigma_{y} R_{i} d\left[1+A_{1}\left(\frac{a}{d}\right)+A_{2}\left(\frac{a}{d}\right)^{2}\right]
$$

186

where

$$
A_{1}=0.066-0.038\left(\frac{\theta}{\pi}\right)-0.960\left(\frac{\theta}{\pi}\right)^{2}
$$

$$
A_{2}=-0.060-1.525\left(\frac{\theta}{\pi}\right)+1.427\left(\frac{\theta}{\pi}\right)^{2}
$$

$M_{L}$ is the plastic limit load for bending and given by

189

$$
M_{L}=4 \sigma_{y} R_{i}^{2} d\left[1+B_{1}\left(\frac{a}{d}\right)+B_{2}\left(\frac{a}{d}\right)^{2}\right]
$$

190

where

$$
B_{1}=0.074-0.169\left(\frac{\theta}{\pi}\right)
$$

191

$$
B_{2}=-0.086-1.013\left(\frac{\theta}{\pi}\right)
$$

192

When the axial tension is constant, the plastic limit bending moment can be expressed as follows

193

$$
M_{L}^{N}=4 \sigma_{y} R_{m}^{2} d \frac{M_{L}}{M_{L}^{\prime}}\left\{\sin \left[\frac{\pi}{2}\left(1-\frac{a}{d} \frac{\theta}{4}-\frac{N}{2 \pi \sigma_{y} R_{m} d} \frac{N_{L}^{\prime}}{N_{L}}\right)\right]-\frac{a}{d} \frac{f(\theta)}{2 \theta}\right\}
$$

194 When the axial tension increases linearly with the bending moment, let the ratio of axial tension 195 to bending moment be defined as follows

$$
\xi=\frac{\sigma_{a}}{\sigma_{b}}=\frac{N\left(R_{o}^{2}+R_{i}^{2}\right)}{4 M R_{o}}
$$
Substituting Equation (16) into Equation (12), the plastic limit bending moment can be obtained as follows

$$
M_{L}^{N}(\xi)=4 \sigma_{y} R_{m}^{2} d \frac{M_{L}}{M_{L}^{\prime}}\left\{\sin \left[\frac{\pi}{2}\left(1-\frac{a}{d} \frac{\theta}{4}-\frac{4 M_{L}^{N} R_{o} \xi}{2 \pi \sigma_{y} R_{m} d\left(R_{o}^{2}+R_{i}^{2}\right)} \frac{N_{L}^{\prime}}{N_{L}}\right)\right]-\frac{a}{d} \frac{f(\theta)}{2 \theta}\right\}
$$


whereas the corresponding plastic limit tension is as follows

$$
N_{L}^{M}(\xi)=\frac{4 M_{L}^{N} R_{o} \xi}{R_{o}^{2}+R_{i}^{2}}
$$

202 It should be noted that $M_{L}^{N}$ in Equation (17) cannot be solved analytically and iterative methods

203 such as Newton' method are needed.

204 Elastic Fracture Toughness for Circumferential External Cracks. For circumferentially 205 cracked pipes under constant axial tension and varying bending moment, substitute Equations 206 (11) and (15) into Equation (5) and it becomes

$$
\begin{gathered}
\frac{K_{r} K_{I C} \sqrt{\pi Q}\left(R_{O}^{4}-R_{i}^{4}\right)}{\sqrt{a}}-N\left(R_{O}^{2}+R_{i}^{2}\right) F_{I}(N)=4 M R_{O} F_{I}(M) \\
L_{r}=\frac{M}{M_{L}^{N}}=\frac{M}{4 \sigma_{y} R_{m}^{2} d \frac{M_{L}}{M_{L}^{\prime}}\left\{\sin \left[\frac{\pi}{2}\left(1-\frac{a \theta}{d 4}-\frac{N}{2 \pi \sigma_{y} R_{m} d N_{L}}\right)\right]-\frac{a f(\theta)}{d 2 \theta}\right\}}
\end{gathered}
$$

208 In Equation (19), by dividing the first equation about $K_{r}$ by the second equation about $L_{r}, L_{r}$ can 209 be expressed as follows

$$
L_{r}=\frac{K_{r} K_{I C} \sqrt{\pi Q}\left(R_{O}^{4}-R_{i}^{4}\right)-\sqrt{a} N\left(R_{o}^{2}+R_{i}^{2}\right) F_{I}(N)}{4 R_{o} \sqrt{a} F_{I}(M)\left\{4 \sigma_{y} R_{m}^{2} d \frac{M_{L}}{M_{L}^{\prime}}\left\{\sin \left[\frac{\pi}{2}\left(1-\frac{a \theta}{d 4}-\frac{N}{2 \pi \sigma_{y} R_{m} d N_{L}}\right)\right]-\frac{a f(\theta)}{d 2 \theta}\right\}\right\}}
$$

211 By solving Equations (6) and (20) simultaneously, the critical value of $K_{r}$ at the fracture, denoted 212 as $K_{r c}$, can be derived as follows

$$
K_{r c}=\left(1+0.5\left(\frac{K_{r c} K_{I C} \sqrt{\pi Q}\left(R_{O}^{4}-R_{i}^{4}\right)-\sqrt{a} N\left(R_{o}^{2}+R_{i}^{2}\right) F_{I}(N)}{4 R_{o} \sqrt{a} F_{I}(M)\left\{4 \sigma_{y} R_{m}^{2} d \frac{M_{L}}{M_{L}^{\prime}}\left\{\sin \left[\frac{\pi}{2}\left(1-\frac{a \theta}{d 4}-\frac{N}{2 \pi \sigma_{y} R_{m} N_{L}}\right)\right]-\frac{a f(\theta)}{d 2 \theta}\right\}\right\}}\right)^{2}\right)^{-0.5}
$$

$$
\left[0.3+0.7 \exp \left(-0.65\left(\frac{K_{r c} K_{I C} \sqrt{\pi Q}\left(R_{O}^{4}-R_{i}^{4}\right)-\sqrt{a} N\left(R_{O}^{2}+R_{i}^{2}\right) F_{I}(N)}{4 R_{O} \sqrt{a} F_{I}(M)\left\{4 \sigma_{y} R_{m}^{2} d \frac{M_{L}}{M_{L}^{\prime}}\left\{\sin \left[\frac{\pi}{2}\left(1-\frac{a \theta}{d 4}-\frac{N}{2 \pi \sigma_{y} R_{m} d N_{L}}\right)\right]-\frac{N^{\prime}(\theta)}{d 2 \theta}\right\}\right\}}\right)\right]\right.
$$


215 Similarly, for circumferentially cracked pipes under combined axial tension and bending with a 216 constant ratio, the relationship between $K_{r}$ and $L_{r}$ can be expressed as follows

$$
L_{r}=\frac{\sqrt{\pi Q} K_{r} K_{I C}\left(R_{o}^{4}-R_{i}^{4}\right)}{4 \sqrt{a} R_{o}\left[\xi F_{I}(N)+F_{I}(M)\right] M_{L}^{N}(\xi)}
$$

222 where $\left\{\left(1+0.5\left(\frac{\bar{\sigma}}{\sigma_{y}}\right)^{2}\right)^{-0.5}\left[0.3+0.7 \exp \left(-0.65\left(\frac{\bar{\sigma}}{\sigma_{y}}\right)^{6}\right)\right]\right\} \leq K_{r c} \leq 1$.

223 When the critical state of pipe failure is reached, the stress intensity factor $K_{I}$ for the brittle 224 fracture will become the elastic critical limit, i.e., elastic fracture toughness $K_{I C}^{e}$, and $K_{r c}$ becomes 225 the ratio $\alpha$ defined in Equation (2). It follows that $K_{I C}^{e}$ can be expressed as follows

$$
K_{I C}^{e}=\alpha K_{I C}=K_{r C} K_{I C}
$$

227 The plastic portion of the fracture toughness can be determined by subtracting the elastic portion 228 in the total fracture toughness

$$
K_{I C}^{p}=(1-\alpha) K_{I C}=\left(1-K_{r C}\right) K_{I C}
$$

230 From Equations (21), (23) and (24), it can be seen that $K_{I C}^{e}$ is a function of crack geometry, 231 material properties and loading conditions of the cracked pipe. It should be noted that for 232 engineering assessment elastic fracture toughness $K_{I C}^{e}$ is the critical stress intensity factor 
assuming the ductile metal pipe is brittle since the plastic behaviour of the metal pipes has been taken into account in deriving $K_{I C}^{e}$.

\section{VERIFICATION AND DISCUSSIONS}

To verify the derived elastic fracture toughness, ideally experimental results should be employed for comparison. However, a thorough literature review suggests that this is extremely difficult. Therefore, the derived model for elastic fracture toughness is verified indirectly by comparing $K_{r}$ with data available in literature. Miller (1984) and Staat and Vu (2013) summarized the burst test results of pipes with circumferential cracks. A close inspection of the published data from literature reveals that very limited data can be used for verification purposes. Most cracks considered have very low aspect ratios, which are out of the applicable range of the stress intensity factor model, and for some tests, no specific material property data were documented. Schulze et al. (1980) carried out tests on steel pipes with both artificially and fatigue induced circumferential cracks of various lengths. The $K_{r}$ value of the failed cracked pipes is calculated from the derived model, i.e., Equation (19), and the ASME boiler and Pressure code (1974), which was often used to interpret the experimental results of cracked pipes (e.g. Brown and Zybenko 1981). The results are shown in Table 2, from which it can be seen that satisfactory agreement has been achieved. The difference in the results might be caused by different finite element models employed and possible variation in determining and formulating the stress intensity factors $K_{I}$ via non-linear regression respectively, as the same $K_{I C}$ is used.

The results from Raju and Newman (1986) are also used for comparison of the stress intensity factors and good agreement has been achieved with a maximum difference of $5.8 \%$. In addition, the plastic load limit has been verified by comparison with results from finite element fracture analyses (Kim et al. 2003) while Equation (6) has been derived as a lower bound of the failure assessment diagrams obtained based on the reference stress approach (SINTAP 1999), which will provide some safety margin for assessment. 
258 Using the derived model, the structural failure of pipes with circumferential external surface 259 cracks under combined tension and bending can be assessed by Equation (4), in which $K_{I}$ and $K_{I C}^{e}$ 260 are calculated by Equations (11) and (24) respectively.

To demonstrate the application of the derived model for elastic fracture toughness, a pressurized pipe with closed ends under bending is taken as an example. In this case, the axial tension induced by internal pressure $p$ is constant for given internal pressure. Consider a steel pipe with a circumferential external crack of $a / c=0.4, a / d=0.75, d / R_{i}=0.1$ and $a=3$. When the internal pressure $p=10 \mathrm{MPa}, K_{r c}$ is 0.839 at the deepest point after solving Equation (21). From Equation (21), it can be seen that the constant axial tension affects the critical limit of $K_{r}$ at fracture. Figure 2 shows the effect of the internal pressure induced tension force on the elastic fracture toughness. It can be seen that the elastic fracture toughness increases with the increase of the internal pressure of the pipe, which indicates that a higher internal pressure results in a smaller portion of fracture resistance offered by plastic deformation.

Equation (6) can be employed to plot the $K_{r}-L_{r}$ curve for parametric study. For given cracked pipes under combined loading, $K_{r}$ and $L_{r}$ can be determined by Equations (20) or (22) for the deepest and surface points. In the following analyses, only the deepest points are considered. Similar analyses can be carried out for surface points, which are omitted here. From Equations (20) and (22), it can be seen that $K_{r}$ and $L_{r}$ exhibit a linear relationship for given combined loadings. For the case with constant aixal tension and varying bending, the amount of axial force controls the slope of the $K_{r}-L_{r}$ line and the intersection point between the $K_{r}-L_{r}$ line and the $K_{r}$ axis. Take the internal pressure induced constant axial tension for example, as shown in Figure 3 a), increasing the internal pressure will decrease the slope of $K_{r}-L_{r}$ line but will increase the $K_{r}$ value at the intersection. When there is no internal pressure in the pipe, the $K_{r}-L_{r}$ line starts from the origin. However, for the case with a constant ratio of axial tension and bending, the $K_{r}-L_{r}$ line 
282 is similar to the case under single loading, in which the line starting from origin as shown in 283 Figure $3 \mathrm{~b}$ ).

284 Figures 4 and 5 demonstrate the effect of material properties on failure mode at the deepest point 285 for a given cracked pipe under bending and constant axial tension. The fracture toughness varies 286 from $50 \mathrm{MPa} / \sqrt{\mathrm{m}}$ to $110 \mathrm{MPa} / \sqrt{\mathrm{m}}$ as shown in Figure 4 while the yield strength changes from $287250 \mathrm{MPa}$ to $650 \mathrm{MPa}$ as shown in Figure 5. It can be observed that for given pipe and crack 288 geometry, the larger the fracture toughness of the pipe material is, the higher portion of plastic 289 deformation the pipe will endure, which means the pipe will fail in a more plastic manner. This 290 makes sense from both the theoretical analysis and practical observation. Similarly, the larger the 291 yield strength of the pipe material is, the higher portion of the brittle fracture the pipe will offer, 292 which indicates that the pipe will fail in a more brittle way. Again, this is consistent with practical 293 experience.

294 Since the relative crack depth $a / d$ is a critical factor that affects the pipe failure its effect on 295 failure mode has also been investigated. As shown in Figure 6, when the relative depth increases 296 from 0.2-0.8, the pipe material exhibits more brittleness. This can be explained by the amount of 297 materials ahead of the crack front. Larger relative depth means less material to develop plastic 298 deformation. Therefore, the pipe failure will tend to be at a lower level of plasticity.

\section{CONCLUSIONS}

300 An analytical model of elastic fracture toughness for ductile metal pipes with circumferential external surface cracks under combined axial tension and bending has been derived in this paper.

302 The derived elastic fracture toughness is a function of crack geometry, material properties and 303 loading conditions of the cracked pipe. One of the benefits for deriving elastic fracture toughness 304 is that the well established linear elastic fracture mechanics can be used for ductile materials in predicting the fracture failure. It has been found in the paper that the elastic fracture toughness 
306 increases with the increase of the internal pressure of the pipe, which indicates that a higher

307 internal pressure results in a smaller portion of fracture resistance offered by plastic deformation.

308 It has also been found that an increase in fracture toughness and yield strength of the pipe 309 materials will result in a more ductile and brittle pipe failure respectively and that an increase in

310 the relative crack depth will lead to less plastic deformation development in the pipe before

311 failure. It can be concluded that the derived analytical model enables more accurate prediction of 312 fracture failure of ductile metal pipes with circumferential external cracks.

\section{ACKNOWLEDGMENTS}

314 Financial support from Australian Research Council under DP140101547, LP150100413 and 315 DP170102211 is gratefully acknowledged. 
$317 \quad a$

$318 A_{1}, A_{2}$

$319 B_{1}, B_{2}$

$320 \quad c$

$321 d$

$322 f$

$323 \quad F_{I}$

$324 \quad h_{i}$

$325 \quad K_{I}$

$326 \quad K_{I C}$

$327 \quad K_{I C}^{e}, K_{I C}^{p}$

$328 \quad K_{r}$

$329 \quad K_{r c}$

$330 \quad L_{r}$

$331 \quad M$

$332 M_{L}, M_{L}^{\prime}$

$333 N$

$334 N_{L}, N_{L}^{\prime}$

$335 p$

$336 P$

$337 \quad P_{L}$

$338 \quad Q$

$339 R_{i}, R_{o}, R_{m}$

$340 \alpha$ depth of a semi-elliptical surface crack

coefficient functions for plastic limit load for axial tension

coefficient functions for plastic limit load for bending

half-length of surface semi-elliptical crack

wall thickness of a pipe

coefficient functions for plastic limit load

influence coefficient functions for Mode I

coefficients of influence coefficient functions

stress intensity factor for Mode I

fracture toughness for Mode I

elastic and plastic fracture toughness for Mode I

ratio for measuring brittle fracture

critical limit of $K_{r}$

ratio for measuring plastic collapse

bending moment

plastic limit load for bending under Mises and Tresca conditions respectively

axial tension

plastic limit load for axial tension under Mises and Tresca conditions respectively

internal pressure

applied external loading

plastic limit load of cracked pipes

elliptical integral of the second kind

internal, external and mean radii of a pipe

ratio of the elastic fracture toughness to the total fracture toughness 
$341 \theta$

$342 \xi$

$343 \bar{\sigma}$

$344 \sigma_{a}, \sigma_{b}$

$345 \sigma_{y}$

$346 \varphi$

half of total crack angle for circumferential cracks, $\theta=c / R_{m}$

ratio of axial tension to bending moment

uniaxial flow stress

axial stresses induced by axial tension and bending respectively

347

position of a point along the semi-elliptical crack

(347 


\section{REFERENCES}

ABAQUS. (2011), Version 6.11 Documentation, Dassault Systèmes Simulia Corp., Providence, RI, USA.

Ainsworth, R.A., (2003), "7.03-Failure assessment diagram methods”, Comprehensive Structural Integrity, 7, 89-132.

Anderson, T.L., (1991), "Fracture mechanics: fundamentals and applications", CRC Press, Boca Raton, Florida.

ASME Boiler and Pressure Vessel Code, (1974), "In service inspection of nuclear power plant components", section 11, Article A. 3000, p. 117.

ASTM E 1820-01, (2003), "Standard test method for measurement of fracture toughness", Pennsylvania, United States: ASTM International.

Brown, K.R. and Zybenko, B., (1981), "The fracture mechanics of aluminium alloy gas cylinders", Engineering Fracture Mechanics, 15, 1-20.

Burdekin, F.M. and Stone, D.E.W., (1966), "The Crack Opening Displacement Approach to Fracture Mechanics in Yielding Materials", Journal of Strain Analysis, 1, 145-153.

Cabral, M.A. and Kimber, M.J., (1997), "Pipeline fracture experiences in Australia and North America", APIA Symposium on Pipeline Fracture, Sydney, Australia, 10 August.

Dowling A.R. and Townley, C.H.A., (1975), "The effects of defects on structural failure: a twocriteria approach", International Journal of Pressure Vessels and Piping, 3, 77-107.

Harrison, R.P., Loosemore, K. and Milne, I., (1976), “Assessment of the Integrity of Structures Containing Defects," Central Electricity Generating Board report, R/H/R6, London.

Jayadevan, K.R., Østby. E., Thaulow. C., (2004), "Fracture response of pipelines subjected to large plastic deformation under tension", International Journal of Pressure Vessels and Piping. 81, 771-783.

Kim, Y.J., Shim, D.J., Huh, N.S. and Kim Y.J., (2002), "Plastic limit pressures for cracked pipes using finite element limit analyses", International Journal of Pressure Vessels and Piping, $79,321-330$.

Kim, Y.J., Shim, D.J., Huh, N.S. and Kim, Y.J., (2003), "Finite element based plastic limit loads for cylinders with part-through surface cracks under combined loading", International Journal of Pressure Vessels and Piping, 80, 527-540.

Kou, K. P. and Burdekin, F. M. (2006), "Stress intensity factors for a wide range of long-deep semi-elliptical surface cracks, partly through-wall cracks and fully through-wall cracks in tubular members", Engineering Fracture Mechanics, 73, 1693-1710.

Li, C.Q., Fu, G.Y. and Yang, W., (2016), "Stress intensity factors for inclined external surface cracks in pressurized pipes", Engineering Fracture Mechanics, 165:72-86. 
Li, C.Q. and Yang, S.T. (2012), "Stress intensity factors for high aspect ratio semi-elliptical internal surface cracks in pipes", International Journal of Pressure Vessels and Piping, 96$97,13-23$.

MathWorks Inc. (2013), MATLAB R2013b. MathWorks, Inc., Natick, MA, USA.

Mettu, S. R., Raju, I. S. and Forman, R. G. (1992), "Stress intensity factors for part-through surface cracks in hollow cylinders", JSC Report 25685/LESC Report 30124, NASA Lyndon B. Johson Space Center/Lockheed Engineering and Sciences Co. Joint Publication.

Miller, A.G., (1984), "Review of test results for ductile failure pressure of cracked spherical and cylindrical pressure vessels", UK: CEGB.

Milne, I., Ainsworth, R.A., Dowling, A.R. and Stewart, A.T., (1988), "Background to and Validation of CEGB Report R/H/R6-Revision 3", International Journal of Presure Vessels and Piping, 32, 105-196.

Pook, L.P., (1995), “On fatigue crack paths”, International Journal of Fatigue, 17(1), 5-13.

Raju, I.S. and Newman, J.C., (1982), "Stress-intensity factors for internal and external surface cracks in cylindrical vessels", Journal Of Pressure Vessel Technology-Transactions Of The ASME, 104(4), 293-298.

Raju, I.S. and Newman, J.C., (1986), "Stress intensity factors for circumferential surface cracks in pipes and rods", Fracture Mechanics: Seventeenth Volume, ASTM STP (Third Edn), 905, 789-805.

Rajeev, P., Kodikara, J., Robert, D., Zeman, P. and Rajani, B., (2014), "Factors contributing to large diameter water pipe failure", Water Asset Management International, 10(3), 9-14.

Rice, J.R., (1968), "A path independent integral and the approximate analysis of strain concentration by notches and cracks", Journal of Applied Mechanics, 35, 379-386.

Schulze, H.D., Togler, G. and Bodmann, E., (1980), "Fracture mechanics analysis on the initiation and propagation of circumferential and longitudinal cracks in straight pipes and pipe bends", Nuclear Engineering and Design, 58, 19-31.

Staat, M. and Vu, D.K., (2013), "Limit analysis of flaws in pressurized pipes and cylindrical vessels. Part II: Circumferential defects”, Engineering Fracture Mechanics, 97, 314-333.

SINTAP, (1999), "Structural Integrity Assessment Procedures for European Industry-Final Procedure", European Union Project No.BE95-1426,

Yang, S.T., Li, C.Q. and Yang, W., (2016), Analytical model of elastic fracture toughness for steel pipes with internal cracks, Engineering Fracture Mechanics, 153, 50-60.

Zhang, Y.M., Yi, D.K., Xiao, Z.M., Huang, Z.H. and Kumar, S.B., (2013), "Elastic-plastic fracture analyses for pipeline girth welds with 3D semi-elliptical surface cracks subjected to large plastic bending", International Journal of Presure Vessels and Piping, 105-106, 90-102. 
421 1. Values of coefficient $h_{i}$ in Equation (8)

422 2. Comparison of $K_{r}$ for the deepest point between the derived model and those from the ASME 423 boiler and Pressure code (1974)

424 
Table 1 Values of coefficient $h_{i}$ in Equation (8)

\begin{tabular}{ccccc}
\hline \multirow{2}{*}{ Coefficients } & \multicolumn{2}{c}{ Under tension } & \multicolumn{2}{c}{ Under bending } \\
\cline { 2 - 5 } & Deepest & Surface & Deepest & Surface \\
$\boldsymbol{h}_{\mathbf{1}}$ & 1.086 & 0.550 & 0.999 & 0.566 \\
$\boldsymbol{h}_{\mathbf{2}}$ & -0.052 & 0.568 & -0.036 & 0.530 \\
$\boldsymbol{h}_{\mathbf{3}}$ & 2.168 & -0.545 & 2.827 & -0.038 \\
$\boldsymbol{h}_{\mathbf{4}}$ & -3.898 & 2.518 & -5.938 & 0.724 \\
$\boldsymbol{h}_{\mathbf{5}}$ & 1.680 & -1.325 & 2.768 & -0.290 \\
$\boldsymbol{h}_{\mathbf{6}}$ & -1.778 & 0.942 & -2.460 & 0.362 \\
$\boldsymbol{h}_{\mathbf{7}}$ & 3.667 & -2.355 & 5.382 & -0.600 \\
$\boldsymbol{h}_{\mathbf{8}}$ & -1.708 & 1.183 & -2.615 & 0.143 \\
$\boldsymbol{h}_{\mathbf{9}}$ & 0.046 & 0.021 & 0.109 & 0.059 \\
\hline
\end{tabular}

428 
431 Table 2 Comparison of $K_{r}$ for the deepest point between the derived model and those from the 432 ASME Boiler and Pressure code (1974)

\begin{tabular}{cccccccccc}
\hline $\begin{array}{c}\boldsymbol{R}_{\boldsymbol{i}} \\
(\mathbf{m m})\end{array}$ & $\begin{array}{c}\boldsymbol{a} \\
(\mathbf{m m})\end{array}$ & $\frac{\boldsymbol{a}}{\boldsymbol{c}}$ & $\frac{\boldsymbol{a}}{\boldsymbol{d}}$ & $\begin{array}{c}\boldsymbol{K}_{I C} \\
(\mathbf{M P a} / \\
\sqrt{\mathbf{m}})\end{array}$ & $\begin{array}{c}\mathbf{N} \\
(\mathbf{k N})\end{array}$ & $\begin{array}{c}\mathbf{M} \\
(\mathbf{k N m})\end{array}$ & $\begin{array}{c}\boldsymbol{K}_{\boldsymbol{r}}(\text { ASME Boiler and } \\
\text { Pressure code 1974) }\end{array}$ & $\begin{array}{c}\boldsymbol{K}_{\boldsymbol{r}} \text { (Derived } \\
\text { model) }\end{array}$ & $\begin{array}{c}\text { Error } \\
(\%)\end{array}$ \\
\hline $\mathbf{3 3 . 5}$ & 3 & 0.25 & 0.75 & 98 & 0 & 9.1 & 0.84 & & \\
\hline $\mathbf{3 3 . 5}$ & 3 & 0.25 & 0.75 & 98 & 35 & 8.2 & 0.85 & 0.78 & 8 \\
\hline
\end{tabular}

433 


\section{$434 \quad$ LIST OF FIGURES}

435 1. A pipe with a circumferential crack subjected to axial tension and bending

436 2. Effect of internal pressure induced axial tension $\mathrm{N}$ on the $K_{r c}$

437 3. Failure assessment diagram for the deepest point

438 4. Effect of fracture toughness on pipe failure at the deepest point

439 5. Effect of yield strength on pipe failure at the deepest point

4406 . Effect of relative crack depth $a / d$ on pipe failure at the deepest point

441 


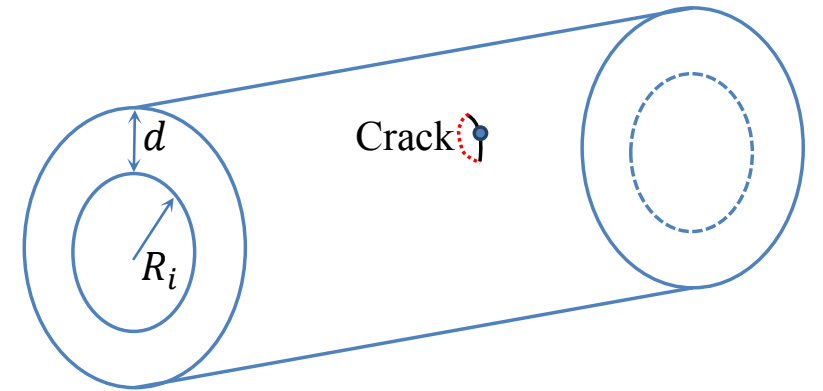

a) Pipe segment

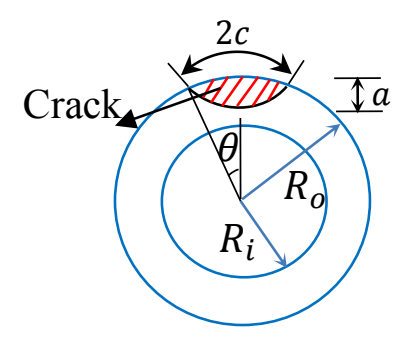

b) Pipe cross section

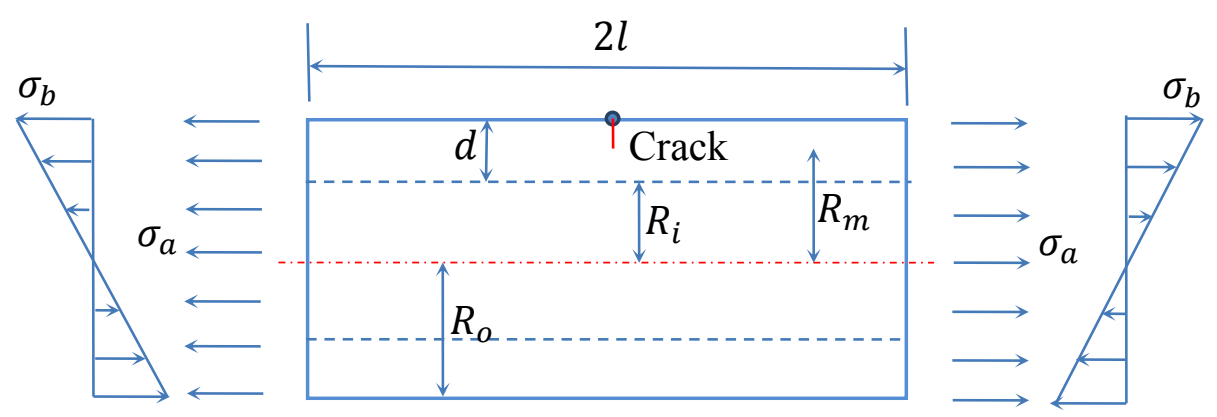

c) Pipe surface area

444

445 Figure 1 A pipe with a circumferential external surface crack subjected to axial tension and 446 bending 


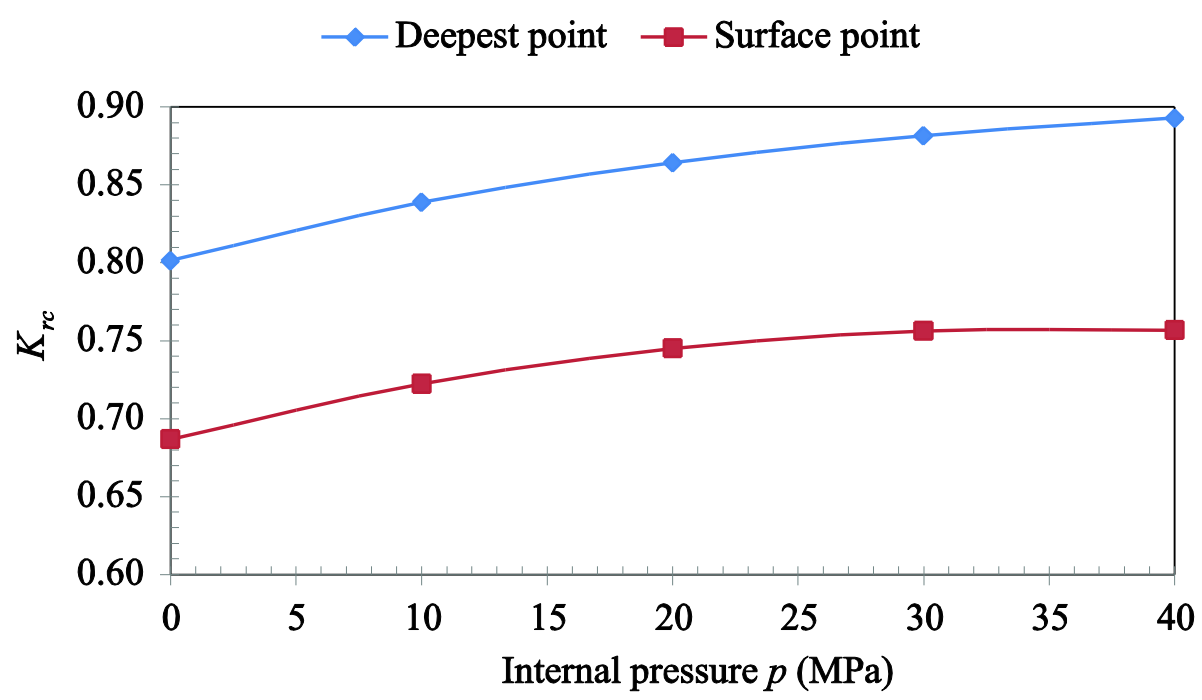

450

451

Figure 2 Effect of internal pressure induced axial tension $N$ on the $K_{r c}$ 


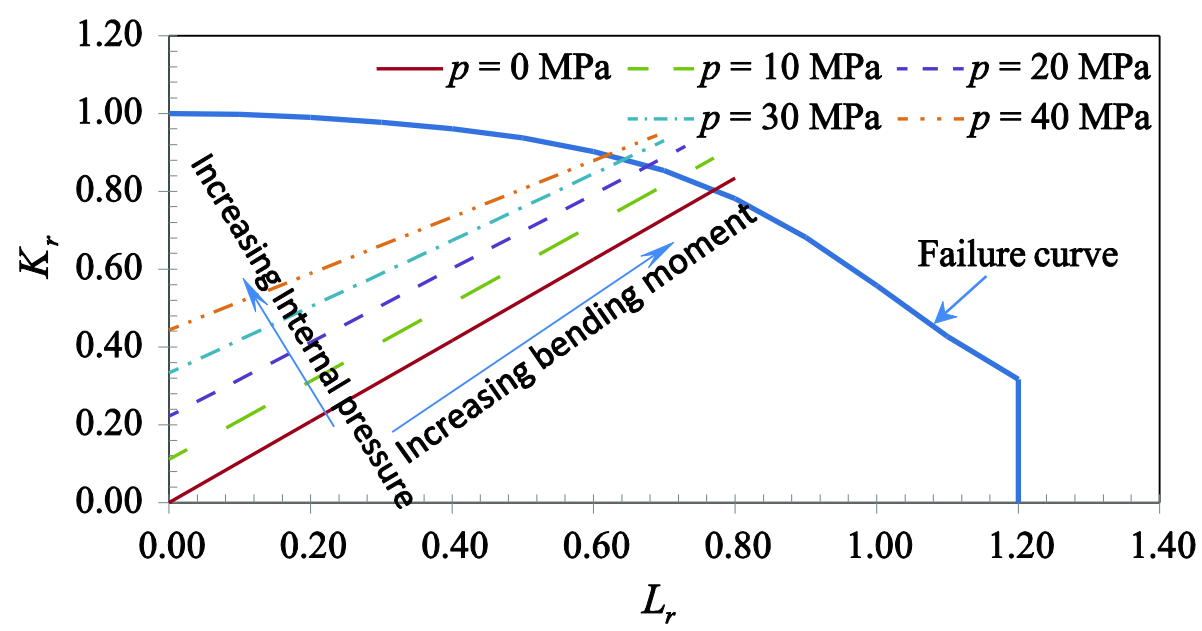

455

456

a) A pressurized pipe under bending

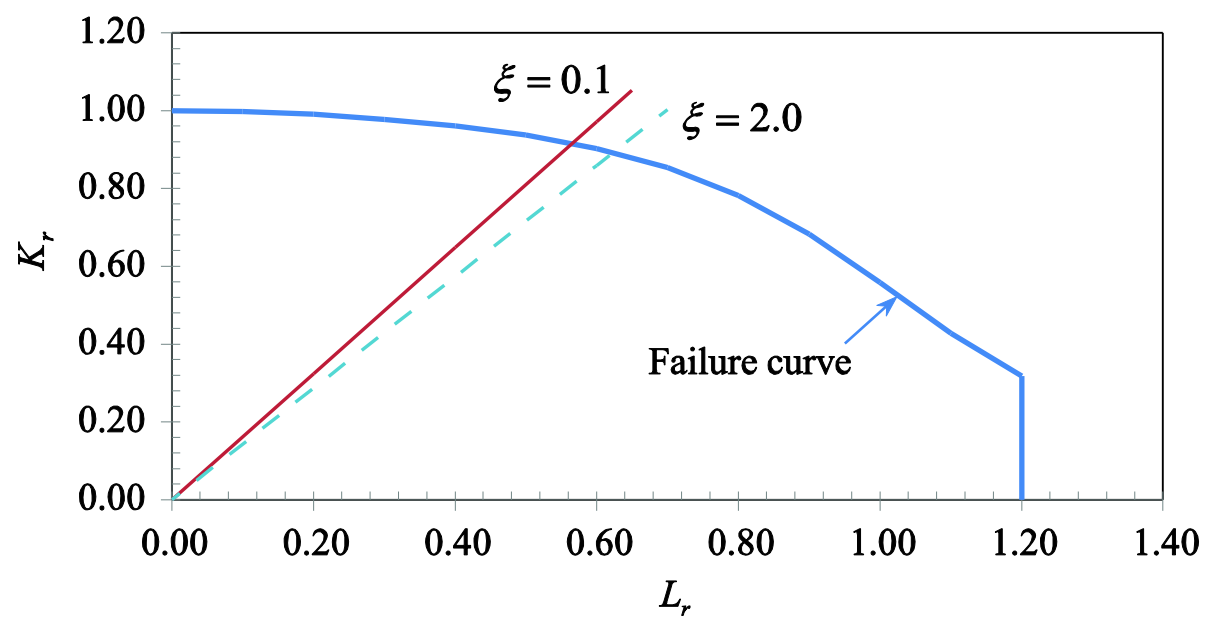

457

458

b) A pipe under combined axial tension and bending with a constant ratio

Figure 3 Failure assessment diagram for the deepest point 


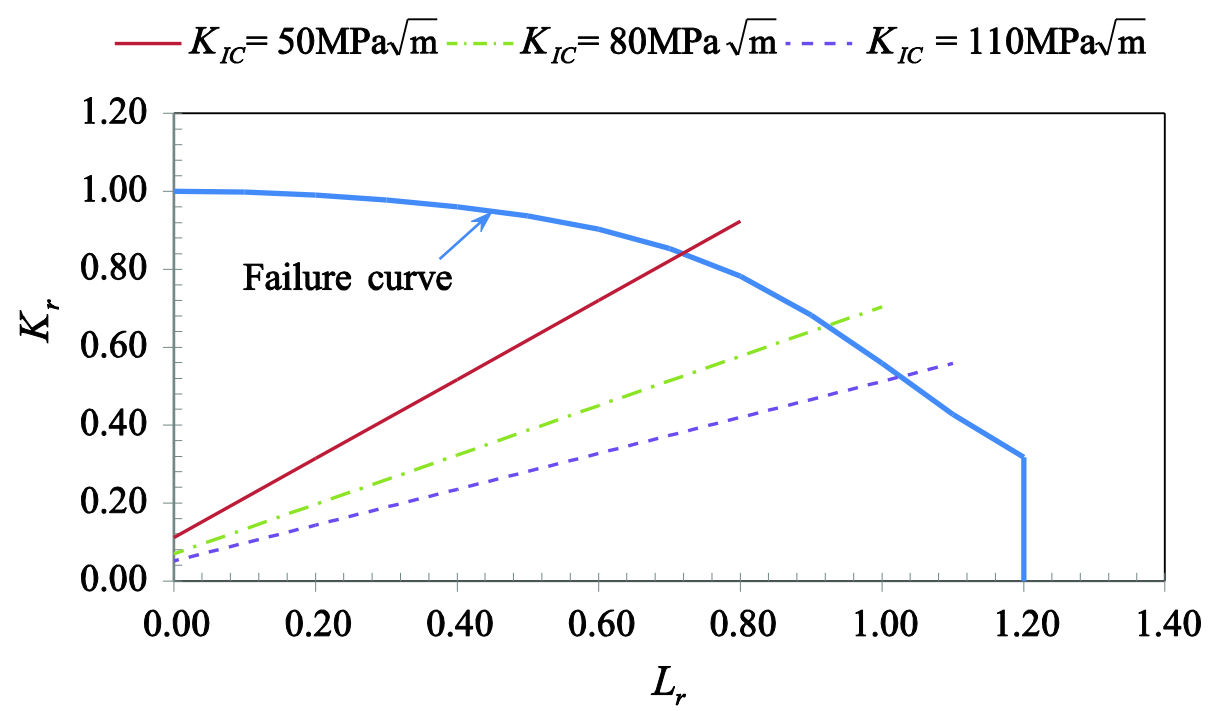

463

464

a) Case with constant axial tension and varying bending

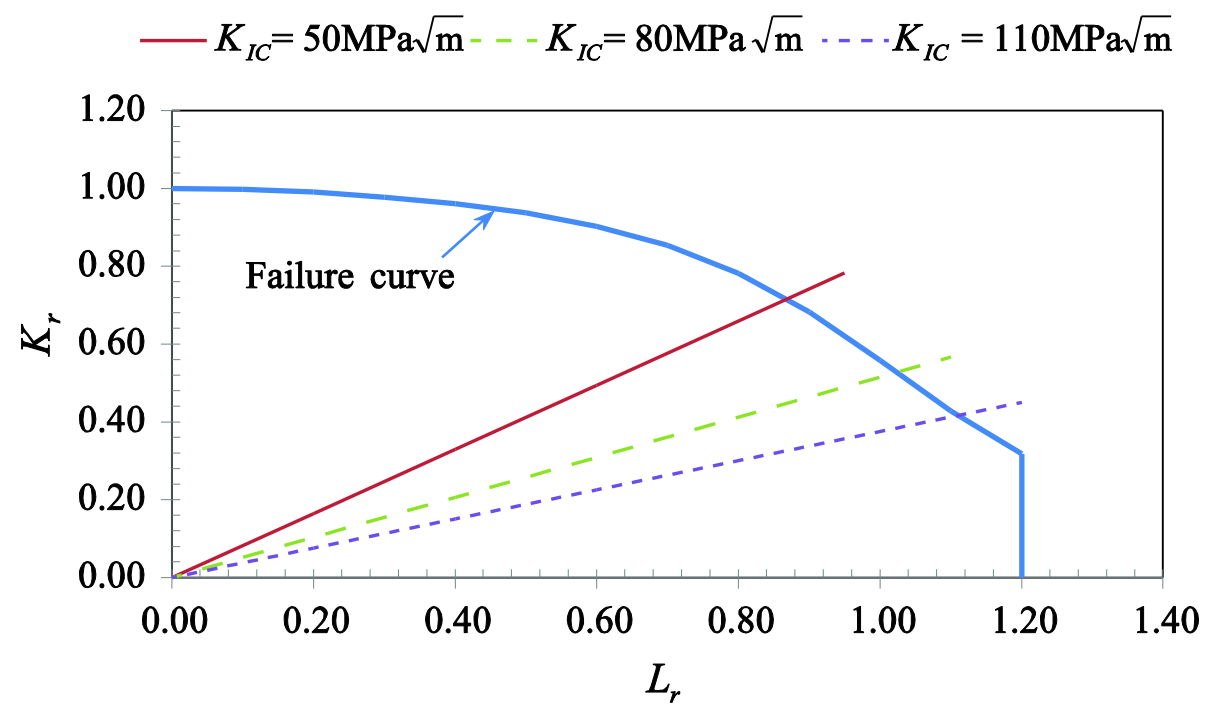

465

466

b) Case with a constant ratio of axial tension to bending

Figure 4 Effect of fracture toughness on pipe failure at the deepest point 


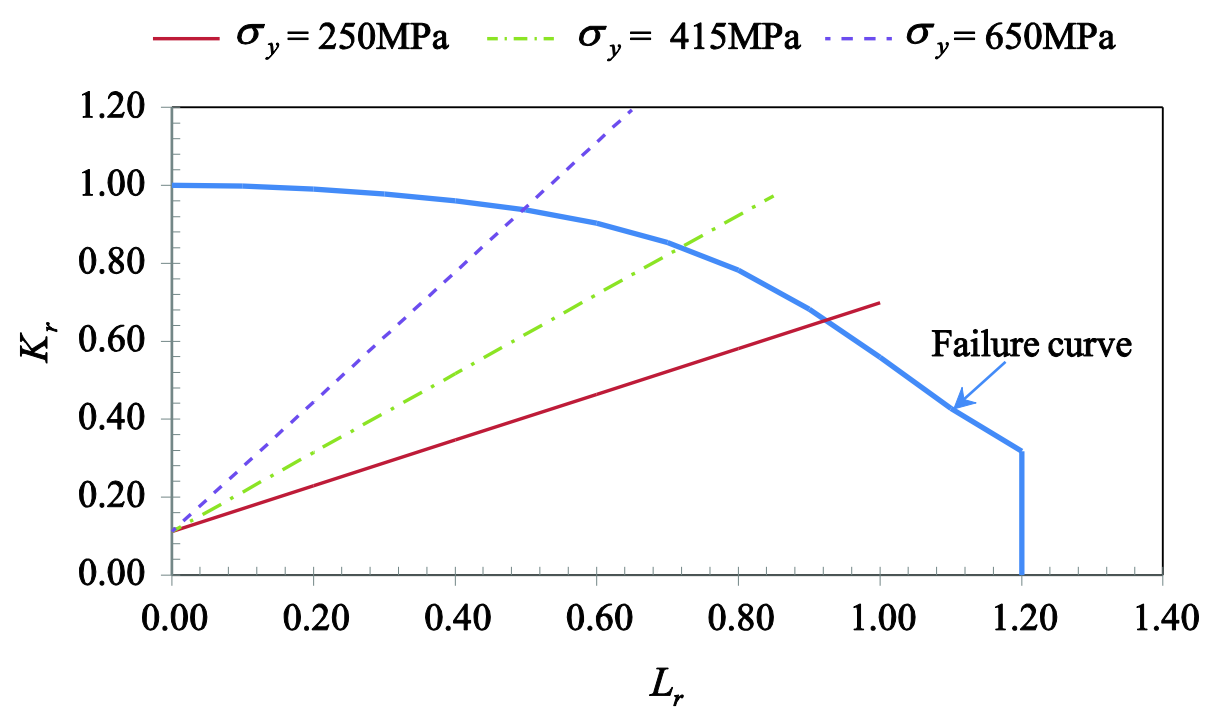

a) Case with constant axial tension and varying bending

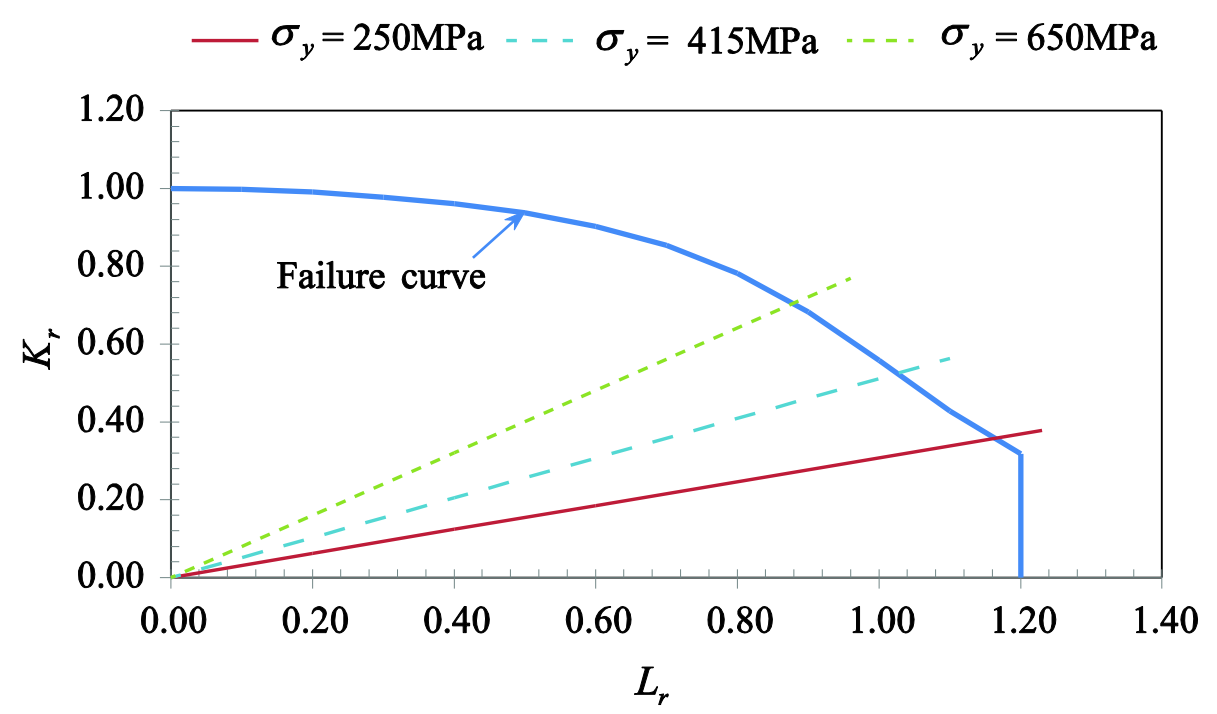

473

b) Case with a constant ratio of axial tension to bending 


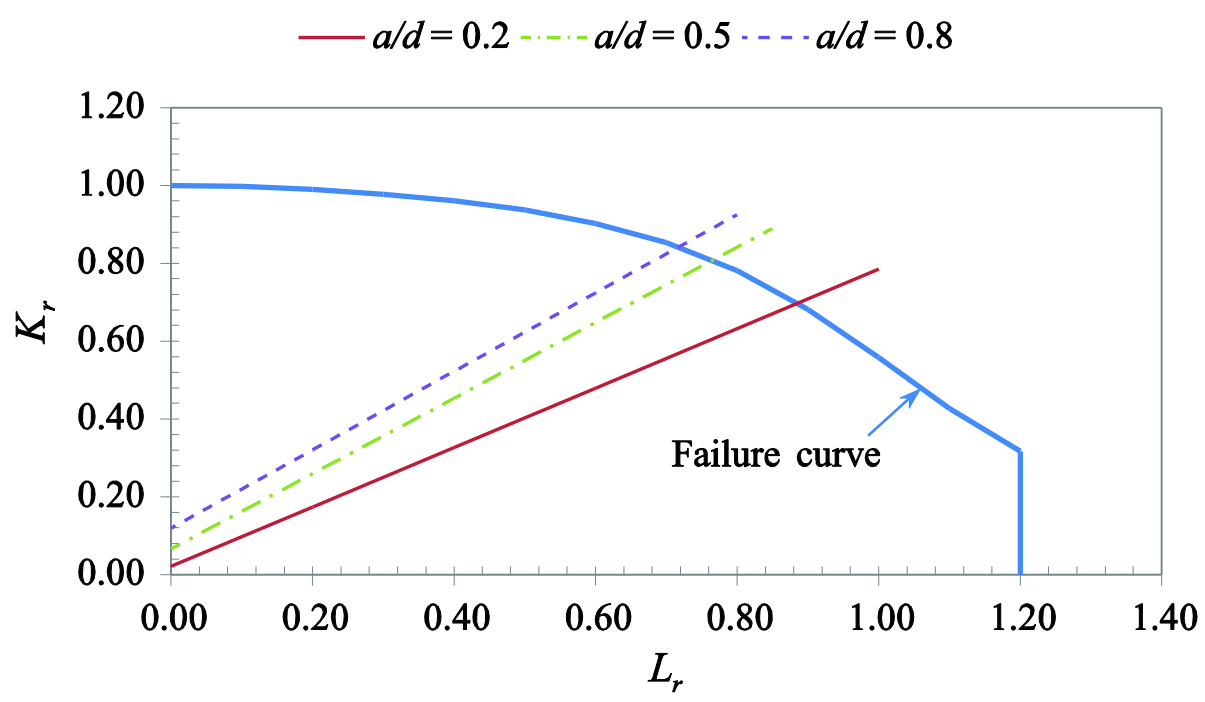

479

480

a) Case with constant axial tension and varying bending

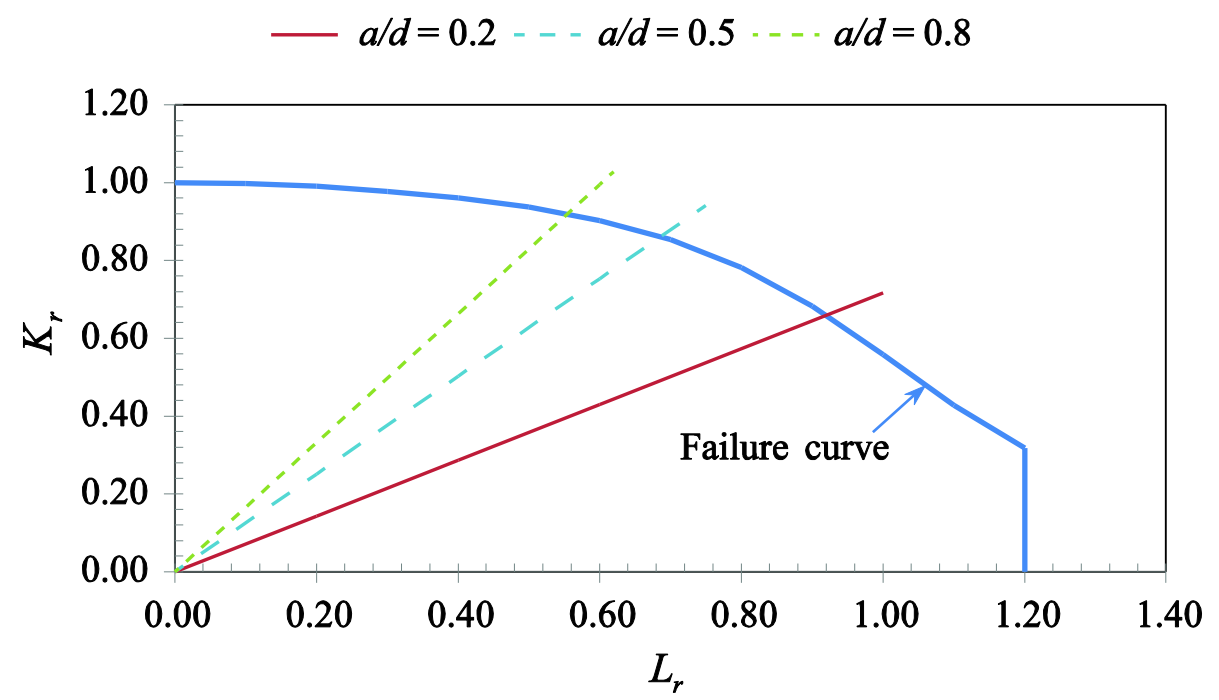

481

b) Case with a constant ratio of axial tension to bending

Figure 6 Effect of relative crack depth $a / d$ on pipe failure at the deepest point 